\title{
A Comprehensive Study on Content based Trademark Retrieval System
}

\author{
Ranjeet Kumar \\ Indian Institute of Information \\ Technology, Deoghat Jhalwa, \\ Allahabad- India.
}

\author{
R.C.Tripathi \\ Indian Institute of Information \\ Technology, Deoghat Jhalwa, \\ Allahabad- India.
}

\author{
M.D.Tiwari \\ Indian Institute of Information \\ Technology, Deoghat Jhalwa, \\ Allahabad- India.
}

\begin{abstract}
World is emerging with trade practices in the global scenario moving mainly towards the brands both for the products and service equally. Most of the Companies are trying to establish the brand name in the market progressively for the global recognition. As a result, Trademarks play today a very critical and important role. Every Company or Financial organization wants a distinguished trademark for brand name and market viability. Trademark registration and its evaluation for distinctiveness is thus becoming very tedious job for registration offices. Millions of trademarks already registered and millions of applications filed for trademarks registration are aggravating the problem of issuing the trademark certificates. There are different techniques and approaches currently in use for distinctness check for trademarks. The present paper gives the overview of the most popular and appreciated image processing techniques and approaches for the trademark distinctness check. Content Based Image retrieval techniques are widely used for that purpose and some other approaches like shape and texture based similarity finding techniques are also used. In the present paper, our approach is to summarize the most widely used techniques for the trademark distinctness check.
\end{abstract}

Keywords: Trademark Retrieval, Content Based Image Retrieval, Information retrieval, Trademark search, trademark distinctness check

\section{INTRODUCTION}

Image processing tools and techniques can be used to solve different problems related to image, text, graphics and color etc. A Trademark can be a combination of text, graphics, image, and colored texture. Based on these, one can divide them in these components for finding the similarity among different trademarks retrieved from the trademark database. The problem for finding the similar trademark has become a challenge because today's world is growing for global economic scenario caused by different trade related practices coming closer to each other at international level. Millions of trademarks being submitted to various trademark offices world over for registration need to have distinctiveness from the existing trademarks as per definitions and trade practices in different countries. Trademark registration with manual searching is very arduous task for the officials and so they need an ICT based practical approach which can show them similar trademarks in the existing database to decide whether to grant the new query trademark a registration or not?
Most of the recent techniques used for the image retrieval have mainly utilized the features like color, texture, shape etc. They used existing CBIR technique, i.e. Content Based Image Retrieval Systems to retrieve the images based on visual features like texture, color, shape etc [1]. In this technique extraction of color feature using the color histogram technique is utilized. It also considers the shape feature because it is an important feature in CBIR applications. Many techniques or approaches have been utilized for the image retrieval, some of which are based on improved pattern matching algorithms. Some others take a much broader approach like searching just from the text files. Some are based on shape and color feature and some have attempted morphological pattern based image matching and retrieval using a database. A shape based technique introduced for the logo retrieval reported in a paper [2] is also inadequate to solve the problem amicably. The main objective of the current paper is to summarize the most widely accepted techniques and approaches for the optimum solutions for the similar trademarks retrieval.

\section{EXISTING TECHNIQUES FOR TRADEMARK RETRIEVAL}

Since last 15 years, there are many techniques developed which are widely accepted for the trademark image retrieval systems Trademark, STAR, ARTISAN are three of the most prominent trademark image retrieval systems. In these three systems, different methodologies and approaches have been used. The TRADEMARK system uses graphical feature vectors (GF-vector) to interpret the image content automatically and calculate the similarity based on human perception [3]. The STAR system adopts mainstream content-based image retrieval (CBIR) techniques. The techniques adopted in this system include the Fourier descriptors, grey level projection and moment invariants. The STAR system works by considering both shape components and the spatial layout of an image [4]. However, the recognition of some perceptually significant components have been considered to be too difficult to be done by an automated process [5]. Therefore, to a certain extent, manual operation is needed for the segmentation of some abstract trademark images. The ARTISAN system, however, introduces an innovative approach that incorporates principles derived from Gestalt psychology to cope with device-only marks which consist of some abstract geometric designs [6].

These three techniques are widely used for trademark image retrieval and logo image retrieval. Some other approaches have been used by different researchers [7]. Hussain and Eakins [8] 
used the topological properties of the self organizing map for similarity retrieval from a trademark image database. Cerri et al. [9] utilized geometrical topological tools for describing trademark shapes and matching their similarity based on size functions of the trademarks. Jiang et al. [10] have given a new approach by using the adaptive selection of visual features with different kinds of visual saliencies, including symmetry, continuity, proximity, parallelism and closure property of the trademark images. Hung et al. [11] illustrated the contour and interior region for retrieving similar trademark images. Kim and Kim [12] used Zernike moments as shape descriptor and conducted experiments based on the MPEG-7 core experiment procedure. Improved performance was reported when compared to other methods based on various shape descriptors; however Zernike moments can only describe global shape. In the above approach of Zernike moment, there is a chance of some improvements. So many other approaches have also been tried for the image or Trademark logo retrieval system but their results seem to be of insignificant improvements.

\section{BASIC FACTS ABOUT CONTENT BASED IMAGE RETRIEVAL SYSTEM (CBIR)}

Content Based Image Retrieval in the prior Art has been earlier used by Kato [13], to describe the experiments of automatic retrieval of images from a database by color, texture and shape features. The features used for retrieval can be either primitive or semantic, but the extraction process must be predominantly automatic. Retrieval of images by manually-assigned keywords is definitely not CBIR as the term is generally understood - even if the keywords describe image content. CBIR draws many of its methods from the field of image processing and computer vision, and is regarded by some as a subset of that field. In the CBIR, the search is to analyze the actual contents of the images. The term 'content' might refer to colors, shapes, textures or any other information that can be derived from the image itself.

\subsection{Basic Principle of CBIR System}

In the other text based approaches of the image retrieval systems, CBIR operates with different principle, to retrieve the stored images from a database by comparing features automatically extracted from the images themselves. The common features used are mathematical measures of color, texture or shape. The current CBIR systems, whether commercial or of open source (experimental) operate at this level. A typical system allows users to formulate queries by submitting an example image, though some other may offer alternatives such as selection from a palette or sketch input. The system then identifies those stored images whose feature values match with those of the query image most closely, and displays thumbnails of these images on the screen. It works in two steps. In the first step the process extracts the distinguishable extent of features from the given image. Thereafter, in the second step, matching theses features with database image features is done to produce the results which are visually similar in decreasing extent. The Figure 1 given below shows the flow chart depicting the basic principle of the CBIR system.

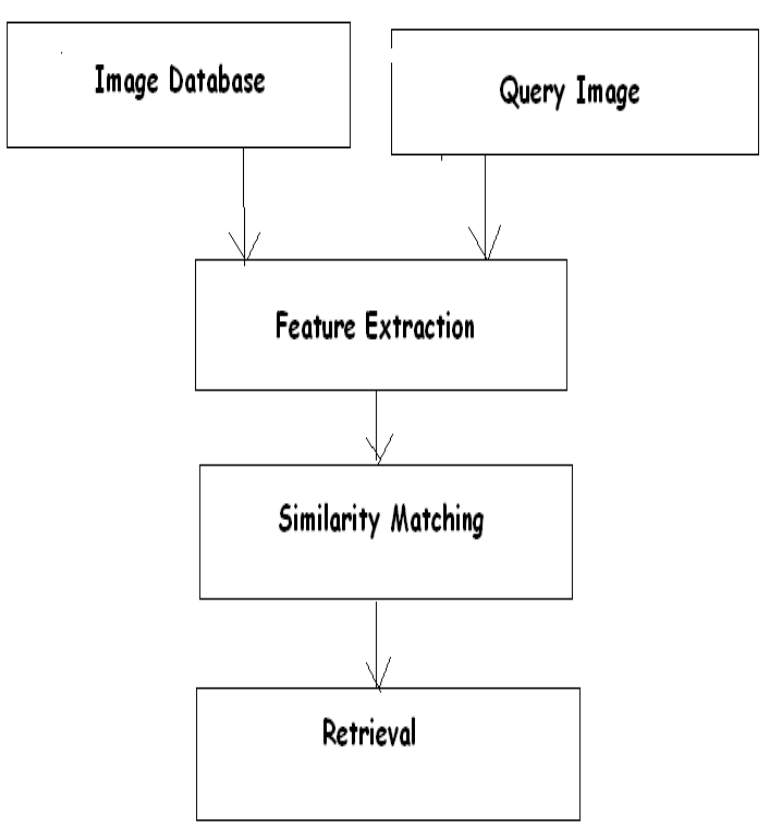

Figure 1. Flow chart of basic Principle of a CBIR System

\subsection{Description of the Content Based Information Retrieval System (CBIR)}

It is important to establish that content-based retrieval does not rely on describing the content of the image in its entirety. A retrieval system presents similar images, similar in some user defined sense. The description of content should serve that goal primarily. The description of contents is described in two stages. First stage defines the image-processing operations that transpose the image data into another spatial data array, and in second stage, methods may be divided on the basis of matching the color, texture, shape or geometry etc.

\subsubsection{Color Image Processing}

Color is one of the most important features of the image that can make possible the recognition of images by humans predominantly. Usually colors are defined in three dimensional color spaces. These could either be Red, Green, and Blue (RGB); Hue, Saturation, and Value (HSV); or Hue, Saturation, and Brightness (HSB). In a CBIR system, color information of images is represented by color histograms. A color histogram is a type of bar graph, where each bar represents a particular color in the color space used. The bars in a color histogram are referred to as bins and they represent the x-axis. The number of bins depends on the number of color frequency bands present in an image. The y-axis denotes the number of pixels there are in each bin.

\subsubsection{Shape Image Processing}

In the Shape Image Processing, all properties are collected that capture conspicuous geometric details in the image. Shape may be defined as the characteristic surface configuration of an object. In objects distinguishing, one also considers the outline of the surroundings of the object. It can be represented in two categories.

Boundary based - Boundary-based shape representation only uses the outer boundary of the shape. This is done 
by describing the considered region using its external characteristics; i.e. the pixels along the object boundary

Region Based- Region-based shape representation uses the entire shape region by describing the considered region using its internal characteristics; i.e. the pixels contained in that region

Figure 2 gives the example of the above two categories which shows the boundary based and region based categories.

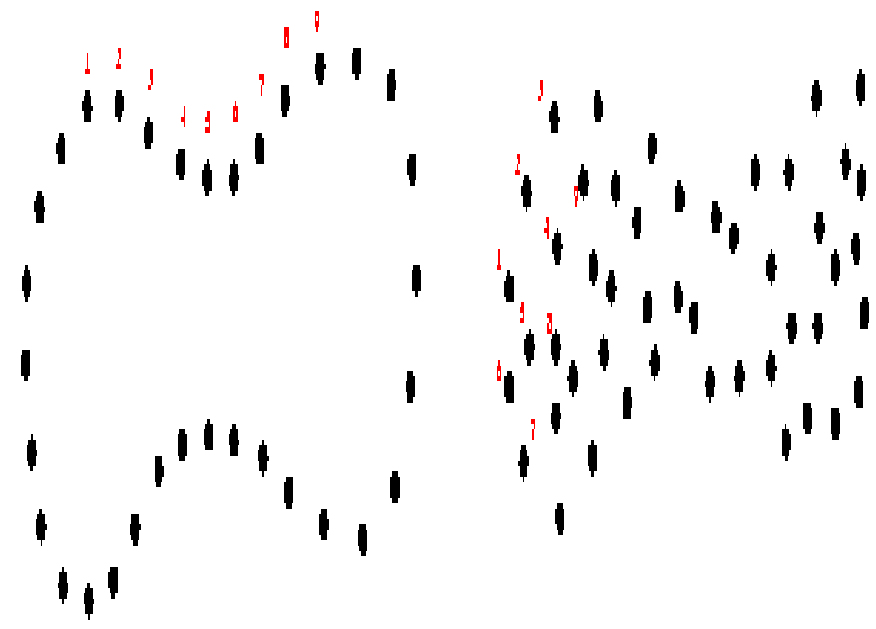

Figure 2. shows the selection of shape based on a) the Boundary and, b) the Region

For the shape feature representation, different mathematical models are used like Boundary based Polygon Models, Fourier Descriptors, Splines, Curvature Model etc. and Region is based on Superquadrics, Implicit Polynomials etc.

\subsubsection{Texture Image Processing}

In computer vision, texture is defined as all what is left after color and local shape have been considered or it is defined by such terms as structure and randomness. Many common textures are composed of small textons usually too great in number to be perceived as isolated objects. The elements can be placed more or less regularly or randomly. They can be almost identical or subject to large variations in their appearance and pose. In the context of image retrieval, research is mostly directed toward statistical or generative methods for the characterization of patches. Basic texture properties include the Markovian analysis, dating back to Haralick in 1973, and generalized versions thereof [14], [15].

Texture is the innate property of all surfaces that describes visual patterns and each having the properties of homogeneity. It contains important information about the structural arrangement of the surface like clouds, leaves, bricks, fabric, etc. For describing the texture, three principles have been used- i.e. Statistical, Structural and Spectral techniques.

Statistical techniques characterize textures using the statistical properties of the grey levels of the points/pixels comprising a surface image. Typically, these properties are computed using the grey level cooccurrence matrix of the surface, or the wavelet transformation of the surface.
Structural techniques characterize textures as being composed of simple primitive structures called "texels" (or texture elements). These are arranged regularly on a surface according to some surface arrangement rules.

Spectral techniques are based on properties of the Fourier spectrum and describe global periodicity of the grey levels of a surface by identifying high-energy peaks in the Fourier spectrum.

\subsection{Scope and Applications of CBIR System}

In the research of wide context of image retrieval and image search, a wide variety of content-based retrieval methods and systems may be found. The different type of search and retrieval on the patterns and applications influence the results of the whole system, so different types of retrievals have been utilized from time to time.

There are three broad categories of aims and user defined system of the image retrieval [16].

- The broad class of methods and systems which searches from unspecified sources and browsing through a large set of images. The users have no target at the beginning of the search and this type of search is in the category of search of associations. Search of association often implies iterative refinement of the search, the similarity or the examples with which the search was started. In this category, the system typically are high interactive, where the specification may be by sketch or by example images.

- In another type of search, users have the target and this is aim for the typical search category. The search will focus and target the image in mind or searching some specific image. In general, these types of systems are suited to search for stamps, art, industrial components and catalogues.

- $\quad$ Another type of search is category search which targets a search aimed to examine if the query image belongs to a specific class. In this case, user may have an image for query and searching is done for the same class images. In this system, searches are defined by catalogues. A typical application of the category search made for classifying the trademarks. Systems in this category are usually interactive with a domain specific definition of similarity.

\section{CBIR BASED COMMERCIAL AND EXPERIMENTAL SYSTEMS}

In this context, several image retrieval systems are available as commercial packages and experimental suits. Out of these, some are most prominent in the market based on their results in different cases. QBIC or Query By Image Content, The ARTISAN System, VisualSEEK and WebSEEK, NeTra etc. VisualSEEK and WebSEEK were developed by Department of Electrical Engineering, Columbia University. Both the systems support color and spatial location and texture matching.

NeTra is developed by Department of Electrical and Computer Engineering, University of California. It supports color, shape layout and texture matching and it also considers the image 
segmentation part. Similar images are retrieved based on color features, Gabor filter bank based texture features, Fourier descriptor based shape features and spatial location information of segmented image regions in NeTra [17].

\subsection{Commercial Systems}

QBIC or Query By Image Content [18] system is probably the best-known of all image content retrieval systems. It is available commercially either in standalone form, or as part of other IBM products such as the DB2 Digital Library. It offers retrieval by any combination of color, texture or shape - as well as by text keyword. Image queries can be formulated by selection from a palette, specifying an example query image, or sketching a desired shape on the screen. The system extracts and stores color, shape and texture features from each image added to the database. At search time, the system matches appropriate features from query and stored images, calculates a similarity score between the query and each stored image examined, and displays the most similar images on the screen as thumbnails. The latest version of the system incorporates more efficient indexing techniques, an improved user interface, the ability to search grey-level images, and a video storyboarding facility etc.

\subsection{Experimental Systems}

A large number of experimental systems have been developed, mainly by academic institutions, in order to demonstrate the feasibility of new techniques. Many of these are available as demonstration versions on the Web. Some of the best-known are described below.

Photobook: - The Photobook [19] system from Massachusetts Institute of Technology (MIT) has proved to be one of the most influential of the early CBIR systems. Like the commercial systems above, aims to characterize images for retrieval by computing shape, texture and other appropriate features. Unlike these systems, however, it aims to calculate informationpreserving features, from which all essential aspects of the original image can in theory be reconstructed. This allows features relevant to a particular type of search to be computed at search time, giving greater flexibility at the expense of speed. The system has been successfully used in a number of applications, involving retrieval of image textures, shapes, and human faces, each using features based on a different model of the image. More recent versions of the system allow users to select the most appropriate feature type for the retrieval problem at hand from a wide range of alternatives.

\section{SOME BASIC LIMITATIONS OF CBIR SYSTEM}

In the field of CBIR, lots of research has been done and sufficient methodologies have been developed for solving different type of problems of image retrieval and matching with different aspects. A general CBIR research is unlikely to be beneficial, when the constraints are matching and retrieval of image in real time scenario. In the research of Theo Pavlidis on CBIR system, he stated some important facts about the progress of CBIR system which is illustrated below [20]. In general, CBIR methods are based on a generality of CBIR systems, i.e. it should be able to calculate a measure of similarity (or distance) between any pair of images because in a general system any image can be a query and any image can be a member of the database [21]. Given below are the facts illustrated by Theo Pavlidis for different scenarios. (i) The mapping between semantics and image features is well defined. This way the issue of "semantic gap" is put to rest. The representation by features can be made without loss of information, in such a way so that only images with similar interpretation and no others are mapped into the same set of features. (Examples abound in the pattern recognition literature, including OCR and fingerprint recognition.)

(ii) The context is known. For example, we may know that the object of interest occupies a particular area in the image or is close in respect of some other well defined feature. The context can be taken advantage in the design and selection of the features.

(iii) The matching of images requires careful scrutiny. This is a process that humans are not very good at and it is likely that machines can match or even exceed human performance. Medical, industrial, forensic, security, financial as well as other applications seem to offer problems of this type.

(iv) The accuracy requirements are well defined. Herein knowing the relative significance of false matches versus omitted true matches is particularly important.

(v) The computational requirements are well defined. In many cases instant response is not needed. For example, in a medical application, it may take well over an hour or a shift to produce an image, so waiting another hour/ shift to find matches in a database is not particularly onerous. In security applications a fast response is often necessary but in that case the database is proprietary (not a public collection) and it may be organized accordingly for fast retrieval using hardware or software or both.

\section{CONCLUSION}

Content Based Image retrieval (CBIR) is used for wide variety of image searches with different patterns and applications. In the present paper, their basic principles and facts have been illustrated. The different types of commercial and experimental systems and the methodology of CBIR have been elaborated which are widely used in the market and research currently. CBIR techniques have been used for different types of searches where users may be of different types as well as their searches. Researchers are working on the current development for these CBIR systems. The new methodologies are approaching for real time scenario retrieval or searches of images in three main categories. Mentions is made of five properties by Theo which creates a hope of not only solving specific problems, but also gaining insight on what methodologies are most appropriate in the CBIR systems. If necessary to work on a general CBIR, the available approaches may be extended to rely on the search for objects by creating "visual words".

\section{REFERENCES}

[1] M.J.Swain and D.H.Ballard, "Color Indexing," International Journal of Computer Vision, vol. 7, no. 1, pp. 11-32, September 1991

[2] A. K. Jain and A. Vailaya, "Shape-Based Retrieval: A Case Study with Trademark Image Databases," Pattern Recognition, vol. 31, no. 9, pp. 1369-1390, 1998

[3] T. Kato, "Database architecture for content based image retrieval", Proceedings of SPIE Image Storage and Retrieval Systems, Vol, 1662, pp, 112-123, 1992 
[4] J.K. Wu, C.P.Lam, B.M. Mehtre, Y.J. Gao, and A. Narasimhalu, "Content based retrieval for trademark registration", Multimedia Tools Application, vol. 3, no. 3, pp. $245-267,1996$

[5] S. Alwis and J. Austin, "A Novel architecture for trademark image retrieval system", Proceedings of the Challenge of Image Retrieval, British Computer Society, UK, 1998.

[6] J. P. Eakins, M.E. Graham, and J.M. Boardman, "Evaluation of a trademark image retrieval system", Information Retrieval Research, the 19th Annual BCS-IRSG Colloquium on IR Research, 1997

[7] Chia-Hung Wei, Yue Li, Wing Yin Chau, and Chang-Tsun Li, Trademark Image Retrieval Using Synthetic Features for Describing Global Shape and Interior Structure, Elsevier Science Inc, Pattern Recognition, Vol 42, Issue 3 March 2009, pages 386-394

[8] M. Hussain and J.P. Eakins, "Component-based visual clustering using the self-organizing map," Neural Networks, vol. 20, no. 2, pp. 260-273, 2007.

[9] A. Cerri, M. Ferri and D. Giorgi, "Retrieval of trademark images by means of size functions,"Graphical Models, vol. 68, no. 5-6, pp. 451-471, 2006

[10] H. Jiang, C.-W. Ngo and H.-K. Tan, "Gestalt-based feature similarity measure in trademark database," Pattern Recognition, vol. 39, no. 5, pp. 988-1001, 2006.

[11] M.H. Hung, C.H. Hsieh, and C.-M. Kuo, "Similarity retrieval of shape images based on database classification," Journal of Visual Communication \& Image Representation, vol. 17, no. 5, 970-985, 2006

[12] W.-Y. Kim and Y.-S. Kim, “A region-based shape descriptor using Zernike moments, "Signal Processing: Image Communication, vol. 16, no. 1-2, pp. 95-102, 2000.
[13] Kato T (1992) "Database architecture for content-based image retrieval" in Image Storage and Retrieval Systems (Jambardino, A A and Niblack, W R, eds), Proc SPIE 1662, $112-123$

[14] S. Krishnamachari and R. Chellappa, "Multiresolution Gauss-Markov Random Field Models for Texture Segmentation," IEEE Trans. Image Processing, vol. 6, no. 2, 1997

[15] G.L. Gimel'farb and A.K. Jain, "On Retrieving Textured Images from an Image Database," Pattern Recognition, vol. 29, no. 9, pp. 1,461-1,483, 1996

[16] Arnold W.M. Smeulders, Marcel Worring, Simone Santini, Amarnath Gupta, Ramesh Jain, Content-Based Image Retrieval at the End of the Early Years, December 2000, IEEE transactions on pattern Analysis and Machine Intelligence, Vol 22, No 12.

[17] W.Y. Ma and B.S. Manjunath, "NeTra: A Toolbox For Navigating Large Image Databases", IEEE Int. Conf. on Image Processing, 1997, pp. 568-571

[18] Flickner, M et al "Query by image and video content: the QBIC system” IEEE Computer1995, 28(9), 23-32

[19] Pentland, R.W. Picard and S. Sclaroff(1996) "Photobook: Content-Based Manipulation of Image Databases" International Journal of Computer Vision 18(3), 233-254

[20] Theo Pavlidis, Limitations of Content-based Image Retrieval, October 24, 2008, Version of June 2008. http://theopavlidis.com/technology/CBIR/PaperB/vers3.htm

[21] Theo Pavlidis, A Set of Images for Testing CBIR Techniques and a Collection of Results, December 2009 http://theopavlidis.com/technology/CBIR/PaperD/testset.htm 